\title{
Influenza A(H1N1)pdm09 Resistance and Cross-Decreased Susceptibility to Oseltamivir and Zanamivir Antiviral Drugs
}

\author{
Vanessa Correia, ${ }^{1}$ Luis A. Santos, ${ }^{1,2}$ Marta Gíria, ${ }^{2}$ Maria M. Almeida-Santos, ${ }^{3}$ \\ and Helena Rebelo-de-Andrade ${ }^{1,2 *}$ \\ ${ }^{1}$ Department of Infectious Diseases, National Health Institute Doutor Ricardo Jorge, Lisbon, Portugal \\ ${ }^{2}$ Centre for Molecular Pathogenesis-Retrovirus and Associated Infections Unit, Institute of Molecular Medicine and \\ Drug Research Institute (iMed.ULisboa), Faculty of Pharmacy, University of Lisbon, Lisbon, Portugal \\ ${ }^{3}$ Clinical Pathology Service, Laboratory of Microbiology, Virology and Molecular Biology, Hospital of Curry Cabral, \\ Lisbon, Portugal
}

Neuraminidase inhibitors (NAls) oseltamivir and zanamivir are currently the only effective antiviral drugs available worldwide for the management of influenza. The potential development of resistance is continually threatening their use, rationalizing and highlighting the need for a close and sustained evaluation of virus susceptibility. This study aimed to analyze and characterize the phenotypic and genotypic NAls susceptibility profiles of $A(H 1 N 1)$ pdm09 viruses circulating in Portugal from 2009 to 2010/2011. A total of 144 cases of $A(H 1 N 1) p d m 09$ virus infection from community and hospitalized patients were studied, including three suspected cases of clinical resistance to oseltamivir. Oseltamivir resistance was confirmed for two of the suspected cases. Neuraminidase (NA) $\mathrm{H} 275 \mathrm{Y}$ resistant marker was found in viruses from both cases but for one it was only present in $26.2 \%$ of virus population, raising questions about the minimal percentage of resistant virus that should be considered relevant. Cross-decreased susceptibility to oseltamivir and zanamivir $\quad\left(2-4 \quad \mathrm{IC}_{50}\right.$ foldchange) was detected on viruses from two potentially linked community patients from 2009. Both viruses harbored the NA I223V mutation. NA Y $155 \mathrm{H}$ mutation was found in 18 statistical non-outlier viruses from 2009, having no impact on virus susceptibility. The mutations at NA N369K and V241I may have contributed to the significantly higher baseline $\mathrm{IC}_{50}$ value obtained to oseltamivir for 2010/2011 viruses, compared to viruses from the pandemic period. These results may contribute to a better understanding of the relationship between phenotype and genotype, which is currently challenging, and to the global assessment of $A(\mathrm{H} 1 \mathrm{~N} 1)$ pdm09 virus susceptibility profile and baseline level to NAls. J. Med. Virol. 87:45-56, 2015.

(C) 2014 Wiley Periodicals, Inc.

KEY WORDS: $A(H 1 N 1) 2009$ pandemic variant; neuraminidase inhibitors; susceptibility testing; NA H275Y; NA I223V; Portugal

\section{INTRODUCTION}

A(H1N1)2009 pandemic was the first influenza pandemic having the assistance of an extensive preparedness and response planning. Advance stockpiling of antiviral drugs was one of the key measures recommend by World Health Organization (WHO), and neuraminidase inhibitors (NAIs), in particular oseltamivir, were the antiviral drugs of choice for many developed countries [WHO, 2005, 2009a; Meijer

Grant sponsor: Portuguese Calouste Gulbenkian Foundation; Grant sponsor: Portuguese Science and Technology Foundation; Grant numbers: SFRH/BD/48532/2008; SFRH/BD/62676/2009; SFRH/BD/65211/2009.

${ }^{*}$ Correspondence to: Helena Rebelo-de-Andrade, Department of Infectious Diseases, National Health Institute Doutor Ricardo Jorge, Av. Padre Cruz, 1649-016 Lisbon, Portugal.

E-mail: h.rebelo.andrade@insa.min-saude.pt

Accepted 2 May 2014

DOI $10.1002 /$ jmv. 23986

Published online 21 July 2014 in Wiley Online Library

(wileyonlinelibrary.com). 
et al., 2007; Oshitani et al., 2008]. Portugal stockpiled a large amount of oseltamivir phosphate (enough for 2.51 million treatments and 100,000 prophylactic schemes) and a small quantity of zanamivir (enough for 42 treatments). This national stockpile was released on April 24, 2009, immediately after the activation of the National Contingency Plan due to A(H1N1)pdm09 virus emergence [DGS, 2010]. National progression from the containment to mitigation pandemic stage on 21st August and availability of the pandemic vaccine on 26th October required timely national updates on the recommendations for antiviral drug use during 2009 [DGS, 2010].

NAIs were an appropriate choice for A(H1N1)2009 pandemic prevention and control. This new variant of the Orthomyxoviridae family, Influenzavirus A genus, exhibited natural resistance to M2 protein inhibitors and clinical effectiveness of NAIs was reported in observational studies carried out during the pandemic period [CDC, 2009; Dominguez-Cherit et al., 2009; Jain et al., 2009; Pada and Tambyah, 2011]. Also, the emergence of resistant variants was only detected for oseltamivir and limited to sporadic events, largely in association with drug use and patients with an immunocompromised condition [WHO, 2011a]. The first case of oseltamivir resistance was reported by Denmark Health Authorities 3 months after the emergence of the $\mathrm{A}(\mathrm{H} 1 \mathrm{~N} 1) 2009$ variant, demonstrating the importance of national capability for the rapid detection of resistant variants [ECDC, 2009]. Since then and until October 5, 2011, a further 604 cases of oseltamivir resistance were detected globally, all harboring the neuraminidase (NA) H275Y mutation. Person-to-person transmission of oseltamivir-resistant virus was identified during the pandemic period but only in three limited clusters, variable in size, timing and location [WHO, 2010, 2011a]. During the following 2010/2011 season, national surveillance activities carried out at the United Kingdom, Japan, and United States reported an increase in the proportion of $\mathrm{A}(\mathrm{H} 1 \mathrm{~N} 1) \mathrm{pdm} 09$ oseltamivir-resistant virus with no known exposure to the drug, potentially indicating a low-level transmission of resistant virus in the community [Lackenby et al., 2011; WHO, 2011a]. This was confirmed recently, by the identification of a community cluster of 6 A(H1N1)pdm09 oseltamivir and peramivir-resistant viruses (H275Y) with no known association to drug use during the end of 2013 in Japan [Takashita et al., 2014]. Regarding zanamivir, only one case of reduced susceptibility was identified to date, in a virus harboring NA I223R mutation collected from an immunocompromised child [WHO, 2011a].

The aim of this study was to analyze and characterize the NAIs susceptibility profiles of influenza A(H1N1)pdm09 viruses circulating in Portugal, from the 2009 pandemic period to the 2010/2011 winter season and in both community and hospital settings, to NAIs (oseltamivir and zanamivir). Suspected cases of clinical resistance development were also studied.

\section{MATERIALS AND METHODS}

\section{Clinical Specimens and Virus Strains}

Clinical specimens from the upper respiratory tract of patients positive for influenza $\mathrm{A}(\mathrm{H} 1 \mathrm{~N} 1)$ pdm09 virus infection were used. Specimens were provided by two national reference hospitals-Hospital de Curry Cabral (HCC) and Hospital Dona Estefânia (HDE) (Lisbon, Portugal). Specimen collection was performed in hospitalized and in community patients attending the hospital emergency unit, from the 2009 pandemic period (June 2009-January 2010) to the 2010/2011 winter season (January-March 2011).

A total of 216 cases of influenza A(H1N1)pdm09 virus infection, 163 from the pandemic period and 53 from 2010/2011, were selected for study. This includes three suspected cases of clinical resistance identified by the hospital clinicians during 2010/2011 in non-related hospitalized patients that failed to respond to oseltamivir treatment (positive for influenza after $>10$ days of therapy), independently of the timing between symptom onset and initiation of therapy $(<$ or $>48 \mathrm{hr}$ ) (Table I). Clinical specimens from these three suspected cases were directly tested for the presence of the NA H275Y oseltamivir-resistant marker. These and all the other 213 specimens from the remaining selected cases underwent viral isolation in MDCK-SIAT1 cells. A total of $142 \mathrm{~A}(\mathrm{H} 1 \mathrm{~N} 1) \mathrm{pdm} 09$ virus strains were isolated successfully, 116 from the pandemic period and 26 from 2010/2011, and evaluated further for phenotypic antiviral drug susceptibility, using mainly a second or third cell passage isolate. This includes the virus strain isolated from suspected case 2 (A/Portugal/03/2011), that was the only suspected case for which viral isolation was successful (Table I). Fifty of the 142 virus strains tested phenotypically, 44 from the pandemic period and 6 from 2010/2011, were evaluated for genotypic antiviral drug susceptibility by NA and hemagglutinin (HA) sequencing. This comprised all virus strains identified as statistical outliers in the phenotypic evaluation and approximately $25 \%$ of the non-outlier strains (randomly selected).

Overall, 144 of the total selected 216 cases of influenza $\mathrm{A}(\mathrm{H} 1 \mathrm{~N} 1)$ pdm09 virus infection were studied. Two cases were only studied genotypically for the specific presence of $\mathrm{H} 275 \mathrm{Y}$ mutation (suspected cases 1 and 3), while the remaining 142 cases (including suspected case 2) were completely studied through phenotypic and/or genotypic analysis.

\section{Screening Assay for Rapid Detection of NA H275Y Oseltamivir-Resistant Marker}

Viral extraction was performed using the automated extractor EasyMag (bioMérieux, Linda-a-Velha, Portugal) according to the manufacturer's recommendations. The presence of NA H275Y oseltamivirresistant marker was searched by real-time RT-PCR, using a protocol kindly provided by Prof. Martin 
TABLE I. Epidemiological, Clinical, and Laboratorial Information Concerning the Three Cases From 2010/2011 Suspected of Clinical Resistance Development to Oseltamivir

\begin{tabular}{|c|c|c|c|}
\hline Case number & 1 & 2 & 3 \\
\hline \multicolumn{4}{|l|}{ Epidemiological and clinical information } \\
\hline \multicolumn{4}{|l|}{ Patient } \\
\hline Age (years) & 1 & 27 & 61 \\
\hline Gender & Male & Female & Male \\
\hline Underlying risk conditions & $\begin{array}{l}\text { Premature bronchopulmonary } \\
\text { dysplasia (hospitalized } \\
\text { since birth) }\end{array}$ & HIV+; pregnant & Not referred \\
\hline Clinical disease & Severe & $\begin{array}{l}\text { Severe with loss of } \\
\text { the fetus }\end{array}$ & Severe \\
\hline \multicolumn{4}{|l|}{ Influenza antiviral drug treatment } \\
\hline Antiviral drug & Oseltamivir & Oseltamivir & Oseltamivir \\
\hline Starting date & $\begin{array}{l}11 \text { days after the } 1 \mathrm{st} \\
\text { positive rt RT-PCR }\end{array}$ & Not referred & Not referred \\
\hline Evidence of failure & $\begin{array}{l}\text { rt RT-PCR positive after } \\
15 \text { days of treatment }\end{array}$ & Not referred & $\begin{array}{l}\text { rt RT-PCR positive after } \\
10 \text { days of treatment }\end{array}$ \\
\hline $\begin{array}{l}\text { Time between the start of antiviral } \\
\text { therapy and specimen collection }\end{array}$ & 20 days & Not possible to estimate & $\geq 10$ days \\
\hline Clinical outcome & Not referred & Deceased & Not referred \\
\hline Other observations & - & $\begin{array}{l}\text { Initiated treatment } \\
\text { with zanamivir; } \\
\text { from Guinea (Africa) }\end{array}$ & - \\
\hline \multicolumn{4}{|l|}{ Clinical specimen laboratorial results } \\
\hline H275Y oseltamivir-resistant marker & Not present & Present $(\mathrm{Ct}=13)$ & $\begin{array}{l}\text { Present as quasi-species } \\
\quad(\mathrm{Ct}=27 \text { for } 275 \mathrm{Y} ; \mathrm{Ct}=28 \\
\text { for } 275 \mathrm{H}), \text { representing } \\
26.2 \% \text { of the virus } \\
\text { population }\end{array}$ \\
\hline Virus strain isolated & No strain isolated & A/Portugal/03/2011 & No strain isolated \\
\hline
\end{tabular}

Curran (Public Health England [PHE], Addenbrooke's Hospital, Cambridge, UK). These methodologies were performed at HCC.

\section{Quantification of H275Y Oseltamivir-Resistant Quasi-Species}

The proportion of wild-type $275 \mathrm{H}$ and mutant $275 \mathrm{Y}$ virus in oseltamivir-resistant quasi-species was quantified by pyrosequencing. This method was performed at the PHE (Colindale, London, UK), following the standard operating procedure in use [HPA, 2010].

\section{Genotypic Evaluation of Virus Susceptibility to NAIs}

Genotypic evaluation was performed by NA and $\mathrm{HA}$ full-length gene sequencing. RNA extraction was performed with QIAamp Viral RNA Mini Kit (QIAGEN, Hilden, Germany). Sequencing methodology and analysis included the CDC protocol recommended by WHO [2009b] and the use of SeqMan application of Lasergene software v.4.05 (DNASTAR, Inc., Madison, WI), as described previously [Santos et al., 2011; Giria et al., 2012]. MEGA5 software was used for multiple sequence alignment by Clustal W method and for mutational analysis against $\mathrm{A} /$ California/07/ 2009 vaccine strain sequences. N1 numbering was used for NA and the signal peptide sequence was not considered for HA numbering. Phylogenetic analysis included (1) determination of the best-fit model for nucleotide substitution according to Akaike Information Criterion, using jModelTest v.2.1.2 [Guindon and
Gascuel, 2003; Darriba et al., 2012]; and (2) construction of phylogenetic trees by maximum-likelihood method using PhyML 3.0 software available on Seaview multiplatform v.4.4.0. Approximate likelihoodratio tests (aLRT(SH-like)) were used for measuring branch support and subtree-pruning-and-regrafting (SPR) and nearest-neighbor-interchange (NNI) rearrangement operations were considered for tree topology improvement. NA and HA sequences of $\mathrm{A} /$ California/07/2009 vaccine strain and of reference strains were included for comparative analysis. These sequences were retrieved from the Global Initiative on Sharing All Influenza Data (GISAID) EpiFlu ${ }^{\mathrm{TM}}$ Database and from the National Center for Biotechnology Information Influenza Virus Resource. Accession numbers are provided in the phylogenetic trees, after the sequence name. In addition, the accession numbers from the sequences downloaded from GISAID database are listed in Table II, to acknowledge the sequence providers.

\section{Phenotypic Evaluation of Virus Susceptibility to NAIs}

Phenotypic profiling was based on $\mathrm{IC}_{50}$ determination, using the new WHO $\mathrm{IC}_{50}$ fold-change criteria that for influenza A viruses is: fold-change increase $<10$-normal inhibition (NI); 10-100 fold-change increase-reduced inhibition (RI); fold-change increase $>100$ - highly reduced inhibition (HRI) [Hurt et al., 2012; WHO, 2012]. Non-outlier IC $_{50}$ values from A(H1N1) seasonal strains that circulated in Portugal 


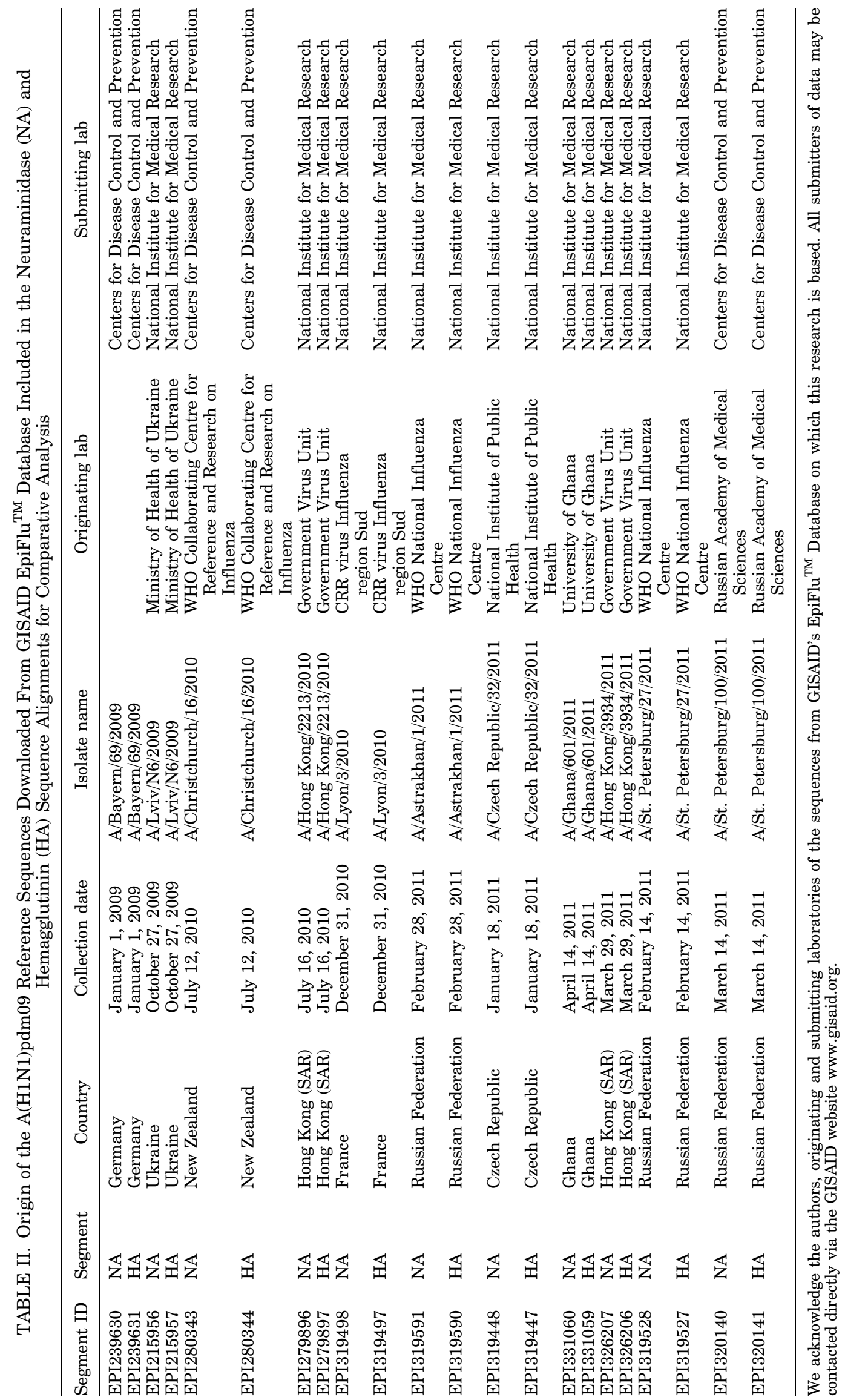


from $2004 / 2005$ to $2008 / 2009$ ( $\mathrm{N}=59$ for oseltamivir; $\mathrm{N}=70$ for zanamivir) [Correia et al., 2010] were used for performing $\mathrm{IC}_{50} \mathrm{~A}(\mathrm{H} 1 \mathrm{~N} 1)$ lineage comparative analysis.

\section{Phenotypic Assay}

Phenotypic testing was performed using the inhouse MUNANA-based $\mathrm{IC}_{50}$ fluorescence assay provided by PHE [HPA, 2006], as described previously in Correia et al. [2010]. Oseltamivir carboxylate and zanamivir were provided through a material transfer agreement by, respectively, F.Hoffmann-La Roche Ltd (Basel, Switzerland) and GlaxoSmithKline (Hertfordshire, UK).

\section{Statistical Analysis}

$\mathrm{IC}_{50}$ values were determined through point-to-point analysis using Microsoft Office Excel 2007. Statistical outliers were identified by determination of upper and lower cut offs, with each one including a mild (1.65 standard deviations [SD] above or below the median, respectively) and an extreme (3 SD above or below the median, respectively) level. Cut offs were determined using Scaled Median Absolute Deviation (SMAD) statistic method [AMC, 2001]. All outlier strains were retested twice and the mean $\mathrm{IC}_{50}$ value was considered for analysis. Median baseline (median without upper and lower outliers) was used for determination of the fold-change thresholds - RI (median $\times 10)$ and HRI (median $\times 100)$, and of the individual fold-changes. Linear regression for time trend analysis was performed using the Microsoft Office Excel 2007 Analysis ToolPak. Cut offs, median baselines, fold-change thresholds and time trends were determined separately for each time period analyzed. Independent sample's $t$ and paired sample's $t$-tests were performed in SPS Statistics software v17.0 and a $P$-value $<0.05$ was considered as statistically significant. All phenotypic data were log-transformed before being used for statistical analysis.

\section{RESULTS}

\section{H275Y Mutation Analysis in Clinical Suspected Resistant Cases}

The presence of NA H275Y mutation was detected in the clinical specimens of both cases 2 and 3 , confirming the suspicion of clinical resistance development to oseltamivir. In the specimen of suspected case 1 , only wild-type $275 \mathrm{H}$ virus was detected by amplification. Detailed results are presented in Table I.

\section{Virus Susceptibility Phenotype to NAIs}

Oseltamivir. A total of $126(88.7 \% ; \mathrm{N}=142)$ $\mathrm{A}(\mathrm{H} 1 \mathrm{~N} 1) \mathrm{pdm} 09$ virus strains, $104(89.7 \% ; \mathrm{N}=116)$ from the pandemic period and $23(88.5 \% ; \mathrm{N}=26)$ from 2010/2011, exhibited an $\mathrm{IC}_{50}$ value within the normal range to oseltamivir (range of values between mild lower and upper cut offs-median $\pm 1.65 \mathrm{SD}$ ). The $\mathrm{IC}_{50}$ values varied from 0.50 to $1.07 \mathrm{nM}$ for pandemic period strains and from 0.69 to $1.21 \mathrm{nM}$ for 2010/2011 strains. The other $15(10.6 \%)$ strains, 12 (10.3\%) from the pandemic period and 3 (11.5\%) from $2010 / 2011$, were identified as statistical outliers. Nine (6.3\%) with $\mathrm{IC}_{50}$ values falling over upper cut offs, 4 at extreme level and 5 at mild level, and 6 (5.2\%) with $\mathrm{IC}_{50}$ values falling under lower cut offs (1 extreme and 5 mild). Figure $1 \mathrm{~A}$ gives information on oseltamivir outlier strains and $\mathrm{IC}_{50}$ fold-changes including all $\mathrm{IC}_{50}$ values obtained for oseltamivir, statistically analyzed and distributed by time. A/ Portugal/03/2011 (isolated from H275Y-confirmed case 2) was the only strain exhibiting a HRI phenotypic profile to oseltamivir, with an $\sim 250$ fold-change increase in the $\mathrm{IC}_{50}$ value $(2010 / 2011 \mathrm{RI}$ threshold $=8.85 ; 2010 / 2011 \mathrm{HRI}$ threshold $=88.50$ ). Despite detection of statistical outliers, all the other 141 strains analyzed showed a NI phenotype to this antiviral drug.

No apparent time grouping was observed for upper and/or lower statistical outliers, with exception of the two upper extreme outliers from 2009 (A/Portugal/17/ 2009 and A/Portugal/82/2009), which are from 2 successive weeks (Fig. 1A). These two outlier strains are from two $\mathrm{A}(\mathrm{H} 1 \mathrm{~N} 1) \mathrm{pdm} 09$ positive cases closely related at the geographic level but without epidemiological link.

Considering non-outlier $\mathrm{IC}_{50}$ values, a significant decreasing trend over time was observed among the 2009 pandemic period values $\left(P=0.00516, \mathrm{R}^{2}=\right.$ 0.074172 , linear regression fit). However, the very low $\mathrm{R}^{2}$ value obtained indicates that time is a poor predictor of $\mathrm{IC}_{50}$ variation. A significant difference was also observed when comparing the non-outlier $\mathrm{IC}_{50}$ values from the two time periods analyzed $(P=0.001$, independent sample's $t$-test).

Zanamivir. For zanamivir, $131(92.3 \%, \mathrm{~N}=142)$ virus strains, $110(94.8 \% ; \mathrm{N}=116)$ from the pandemic period and $21(80.8 \%$; $\mathrm{N}=26)$ from $2010 / 2011$, exhibited an $\mathrm{IC}_{50}$ value within the normal range. Specifically, the $\mathrm{IC}_{50}$ values from pandemic period strains varied from 0.40 to $0.99 \mathrm{nM}$ and those from 2010/ 2011 strains varied from 0.41 to $0.80 \mathrm{nM}$. The other $11(7.7 \%)$ strains, $6(5.2 \%)$ from the pandemic period and $5(19.2 \%)$ from 2010/2011, were identified as statistical outliers. Six $(5.2 \%)$ with $\mathrm{IC}_{50}$ values falling over upper cut offs, one at extreme level and five at mild level, and five (3.5\%) with $\mathrm{IC}_{50}$ values falling under lower cut offs (one extreme and four mild). Figure 1B shows information on zanamivir outlier strains and $\mathrm{IC}_{50}$ fold-changes including all zanamivir $\mathrm{IC}_{50}$ values, statistically analyzed and distributed by time. The strains A/Portugal/17/2009, A/Portugal/82/ 2009, and A/Portugal/07/2011 exhibited to zanamivir the same phenotype displayed to oseltamivir, of upper outlier. Despite detection of statistical outliers, all the $142 \mathrm{~A}(\mathrm{H} 1 \mathrm{~N} 1)$ pdm09 analyzed strains shared a NI phenotypic profile to zanamivir (RI fold-change 


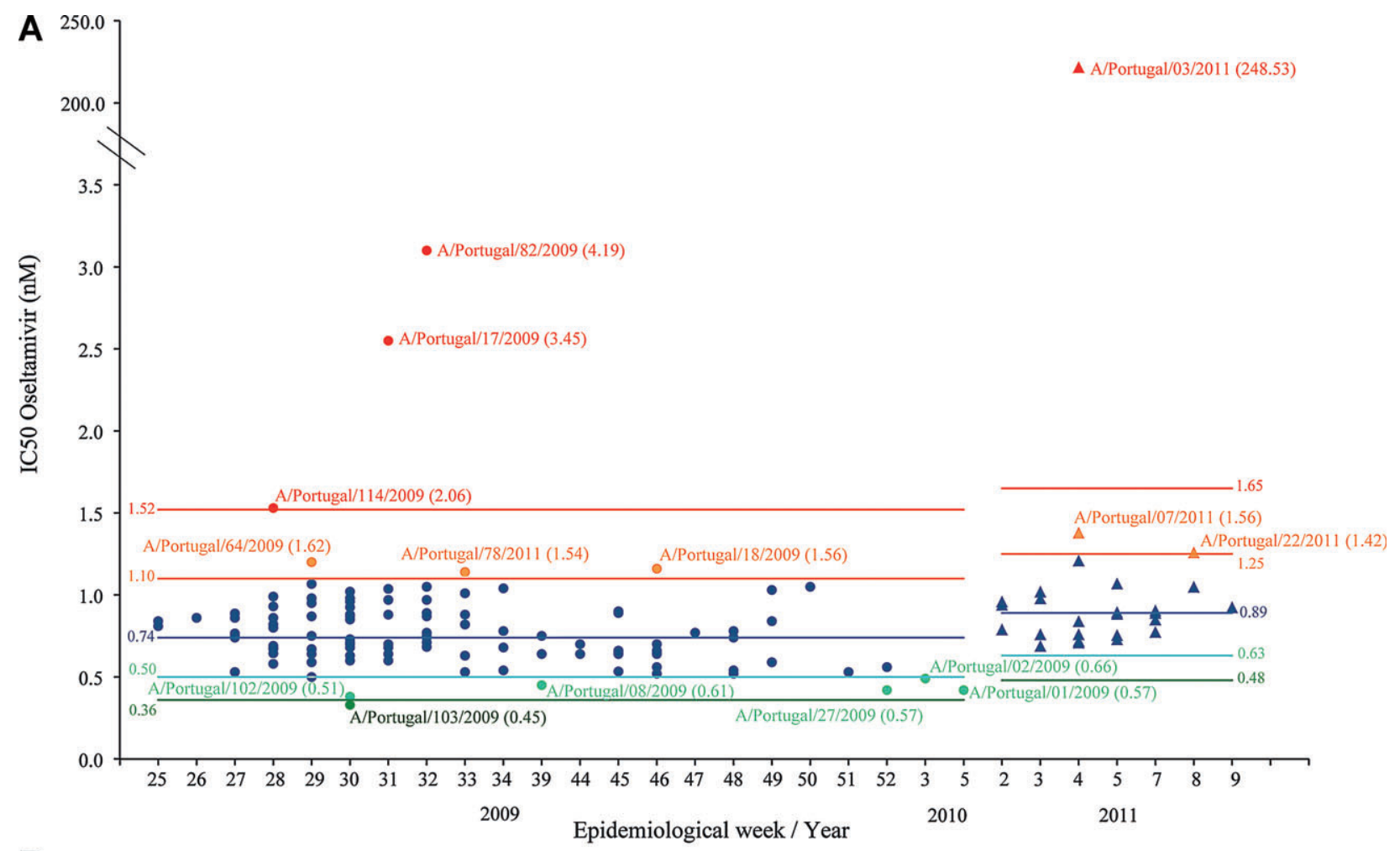

B

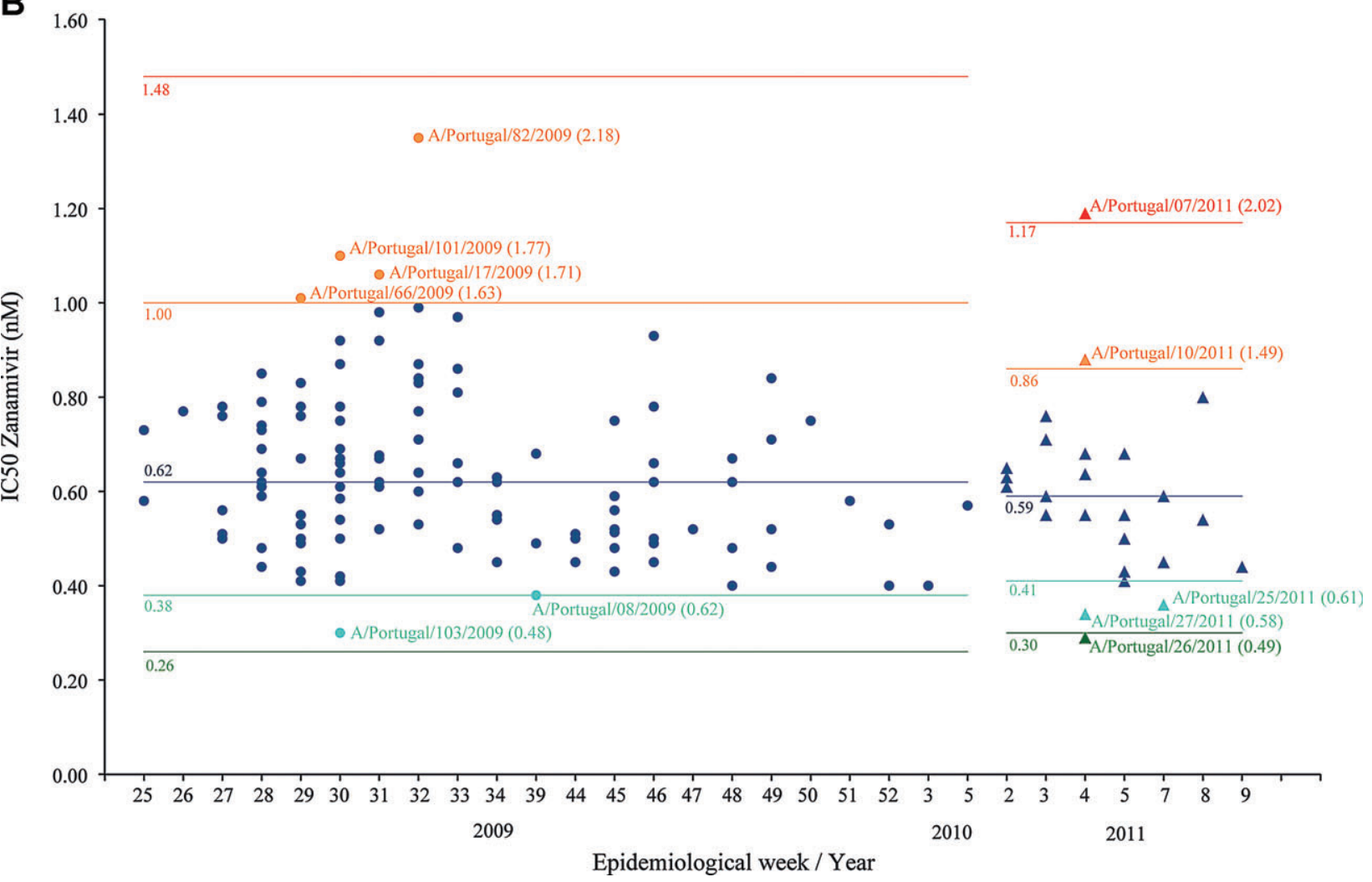

Fig. 1. SMAD statistical analysis of the $\mathrm{IC}_{50}$ values obtained for the $142 \mathrm{~A}(\mathrm{H} 1 \mathrm{~N} 1)$ pdm09 virus strains analyzed phenotypically, 116 from the pandemic period and 26 from the 2010/2011 season, displayed by week of specimen collection, for oseltamivir (A) and zanamivir $(\mathbf{B})$. The $\mathrm{IC}_{50}$ values of virus strains from the pandemic period are coloured in black and those of virus strains from 2010/2011 are coloured in white (colored web version: circles represent the $\mathrm{IC}_{50}$ values of virus strains from the pandemic period and triangles the $\mathrm{IC}_{50}$ values of virus strains from 2010/2011). The $\mathrm{IC}_{50}$ foldchange observed in relation to median baseline is indicated in brackets after the strain designation. 
threshold $=6.20$ [pandemic period] or 5.90 [2010/ 2011]; HRI fold-change threshold $=62.0$ [pandemic period] or 59.0 [2010/2011]).

Time analysis revealed that upper outliers from the pandemic period grouped between weeks 29 and 32 of 2009 , but no epidemiological link was found (Fig. 1B).

Concerning non-outlier $\mathrm{IC}_{50}$ values, a significant decreasing trend over time was detected for 2009 pandemic period values $\left(P=0.00481, \mathrm{R}^{2}=0.071277\right.$, linear regression fit). However, as observed for oseltamivir, the very low $\mathrm{R}^{2}$ value prevents interpretation of the causal relationship between these two variables. No significant difference was observed between the values from the two time periods analyzed ( $P=0.374$, independent sample's $t$-test).

Oseltamivir and zanamivir non-outlier $\mathrm{IC}_{50}$ values differed significantly $(P=0.000$, paired sample's $t$ test). A significant difference was also observed for both NAIs when comparing the non-outlier $\mathrm{IC}_{50}$ values from the $\mathrm{A}(\mathrm{H} 1 \mathrm{~N} 1) \mathrm{pdm} 09$ strains analyzed here with those from $\mathrm{A}(\mathrm{H} 1 \mathrm{~N} 1)$ seasonal strains that circulated at national level from 2004/2005 to 2008/ 2009 ( $P=0.000$, independent sample's $t$-test) (Fig. 2).

A total of $12 \mathrm{~A}(\mathrm{H} 1 \mathrm{~N} 1)$ pdm09 virus strains (8 from the pandemic period and 4 from 2010/2011) were classified as upper statistical outliers for oseltamivir and/or zanamivir and thus selected for further investigation at genotypic level. From these, only A/ Portugal/03/2011 had a known association to drug use, having been isolated from suspected case 2 clinical specimen.

\section{Virus Susceptibility Genotype to NAIs}

NA and HA amino acid mutations found specifically in the sequences of the 12 phenotypic upper outlier strains are indicated and characterized in Table III.

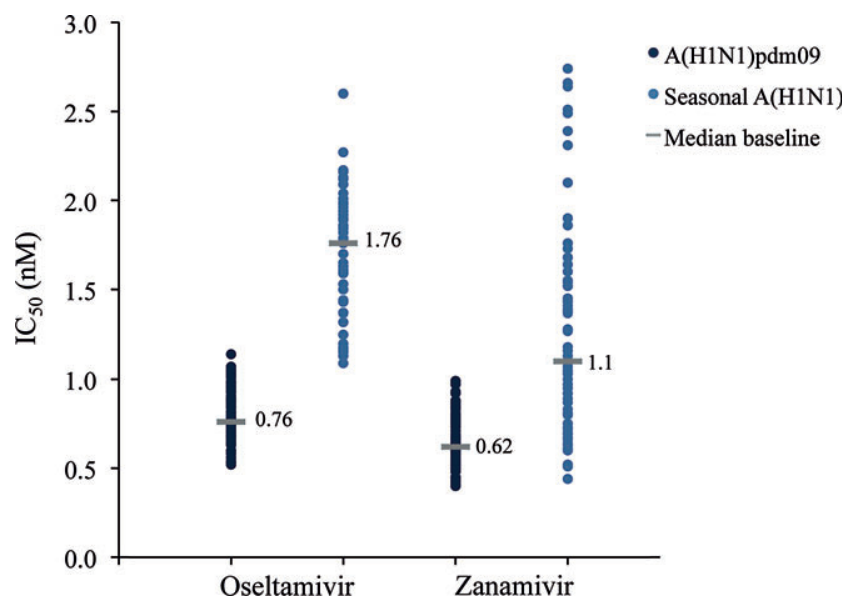

Fig. 2. Comparative analysis of oseltamivir and zanamivir non-outlier $\mathrm{IC}_{50}$ values obtained for the $\mathrm{A}(\mathrm{H} 1 \mathrm{~N} 1) \mathrm{pdm} 09$ virus strains analyzed in this study $(\mathrm{N}=126$ oseltamivir; $\mathrm{N}=132$ zanamivir) and for seasonal $\mathrm{A}(\mathrm{H} 1 \mathrm{~N} 1)$ virus strains previously circulating at national level, from 2004/2005 to 2008/2009 $(\mathrm{N}=59$ oseltamivir; $\mathrm{N}=70$ zanamivir).
NA-specific mutations of oseltamivir extreme outlier strains A/Portugal/17/2019, A/Portugal/82/2009 and A/Portugal/03/2011, have a known reduced susceptibility profile (I223V and $\mathrm{H} 275 \mathrm{Y}$ ). Only A/Portugal/03/ 2011 exhibited specific mutations in the HA sequence, harboring an N156D mutation, located in an antigenic site. Regarding all the other oseltamivir and/or zanamivir outlier strains, no shared mutation or known association to a reduced susceptibility profile were identified. In addition, approximately half of these outliers lacked any specific mutations at NA or HA sequences (Table III).

Mutation NA Y155H, associated with a HRI phenotypic profile to both NAIs in seasonal A(H1N1) subtype [Nguyen et al., 2012], was found in the sequence of 18 non-outlier $\mathrm{A}(\mathrm{H} 1 \mathrm{~N} 1)$ pdm09 strains from 2009 (12.7\%; 18/142), having no apparent impact on NAIs phenotypic susceptibility (Fig. 3A).

Phylogenetic analysis of NA and HA nucleotide sequences revealed two important findings: (1) lack of nucleotide differences between the sequences of the two upper outlier strains A/Portugal/17/2009 and A/ Portugal/82/2009, with pairwise distance calculations confirming the $100 \%$ identity (results not shown); and (2) no clustering among all the other outlier strains, with outlier sequences being closely related to non-outlier sequences from the same time period (Fig. 3A and B).

\section{DISCUSSION}

This study comprised the analysis of $\mathrm{A}(\mathrm{H} 1 \mathrm{~N} 1)$ pdm09 virus collected from community and from hospitalized patients, with and without antiviral drug treatment. Using this integrated approach it was possible to cover the two possible settings where influenza antiviral drug resistance or reduced susceptibility can emerge-spontaneously in the community or associated with drug use.

Oseltamivir resistance was identified for two A(H1N1)pdm09 cases from 2010/2011, both from oseltamivir-treated patients (suspected cases 2 and 3 ). The NA H275Y resistant marker was found in viruses from both cases but for one (suspected case 3) it was only detected in $26.2 \%$ of the clinical specimen viral population. The fact that this percentage of resistant virus could have been sufficient for causing treatment failure raised questions about the minimal percentage of resistant virus that should be considered relevant in quasi-species. For suspected case 2, the NA H275Y mutation was identified in the entire viral population of both clinical specimen and virus isolate (A/Portugal/03/2011 strain). Phenotypic analysis of this virus strain revealed a HRI profile to oseltamivir $\left(\mathrm{IC}_{50}=219.95 \mathrm{mM} ; \sim 250\right.$ fold-change reduction). When compared with H275Y oseltamivirresistant $A(H 1 N 1)$ seasonal strains that circulated previously at national level $\left(\mathrm{IC}_{50}=314.04-1208.84 \mathrm{mM}\right.$; 159-613 fold-change) [Correia et al., 2010], this $\mathrm{A}(\mathrm{H} 1 \mathrm{~N} 1)$ pdm09 strain exhibited a lower $\mathrm{IC}_{50}$ value and 


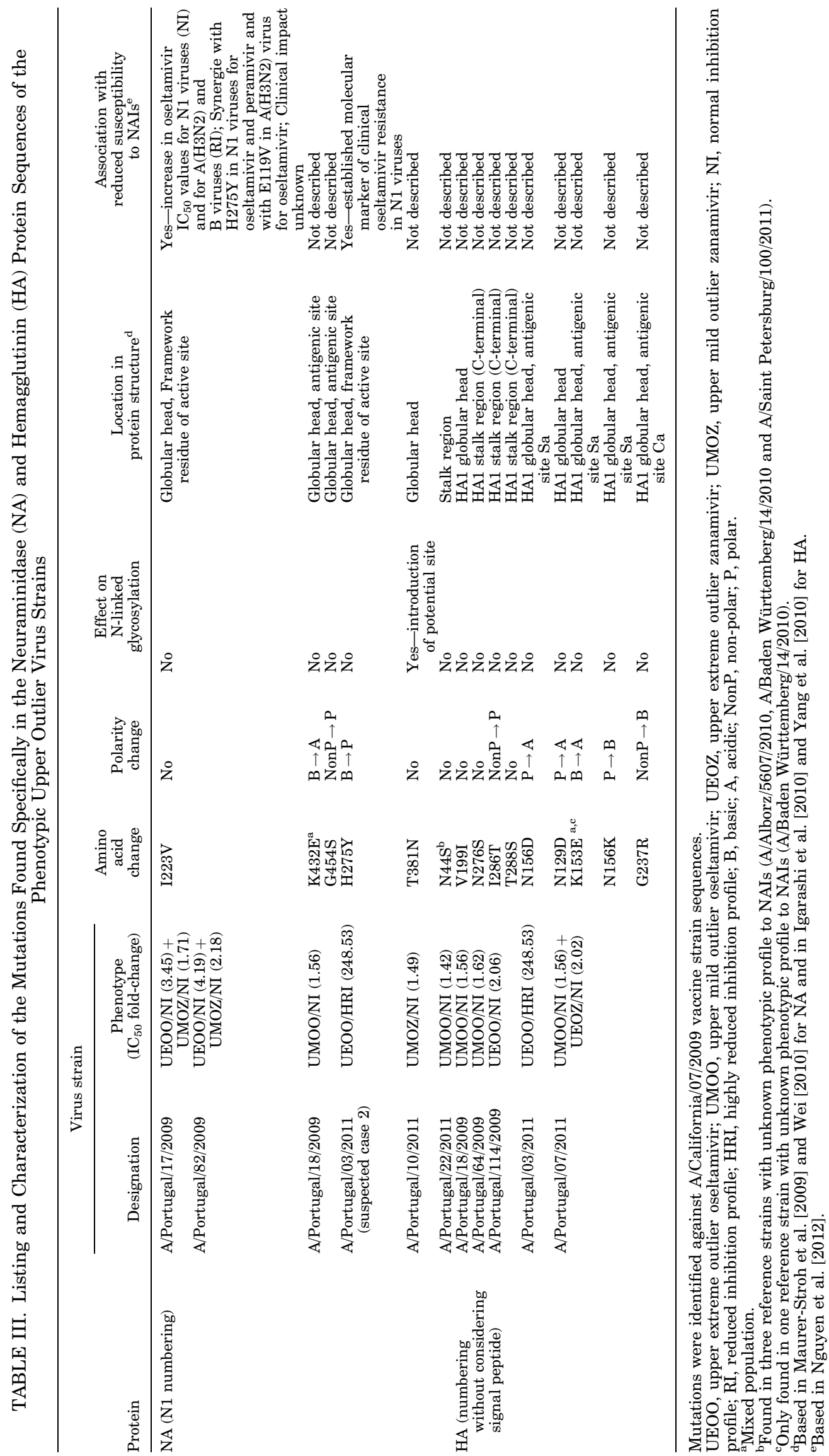




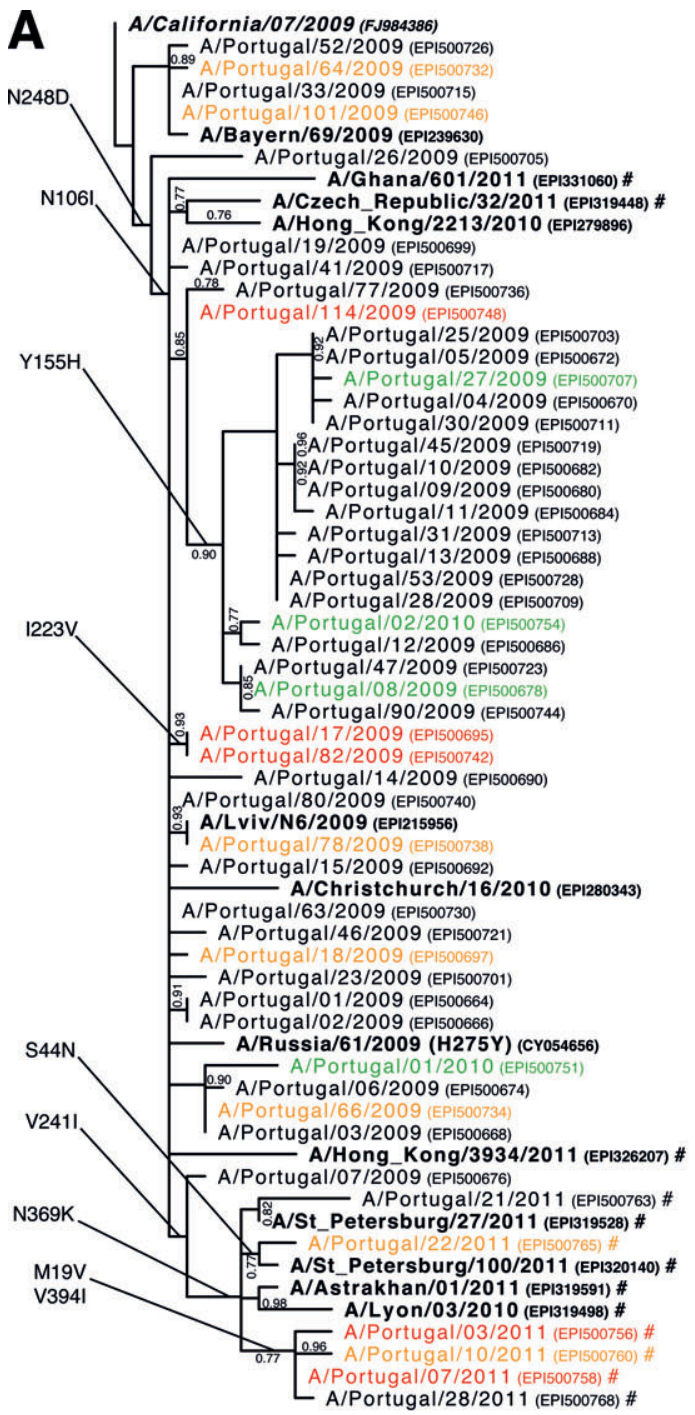

0.0070

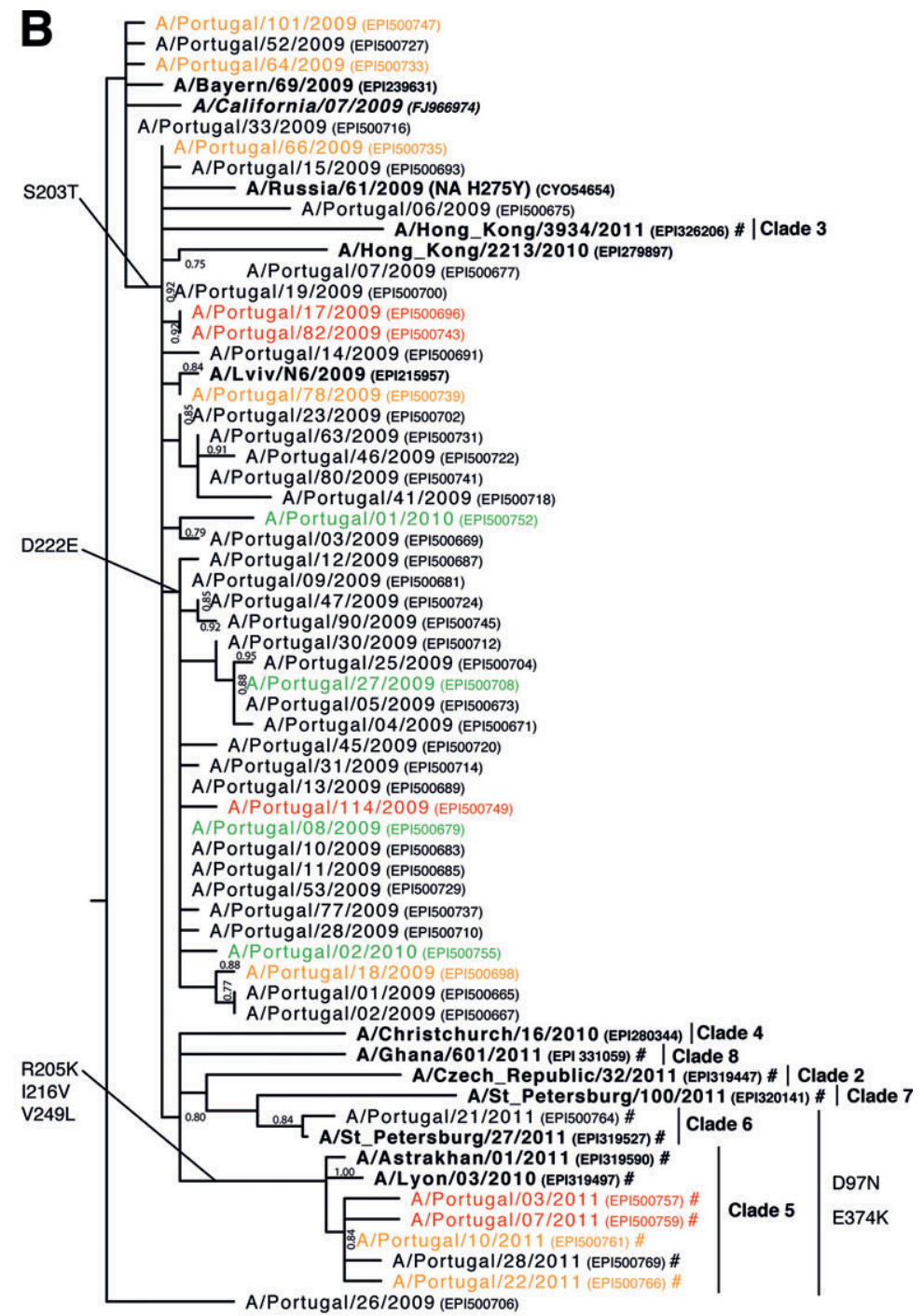

0.0050
Fig. 3. Phylogenetic analysis of the neuraminidase (A) and hemagglutinin (B) full-length nucleotide sequences of the 50 $\mathrm{A}(\mathrm{H} 1 \mathrm{~N} 1) \mathrm{pdm} 09$ virus strains evaluated genotypically, 44 from the pandemic period and 6 from 2010/2011. Nucleotide substitution models GTR $+\Gamma 4$ (General Time Reversible model with a 4category gamma distribution for rate variation among sites) and HKY85+I (Hasegawa-Kishono-Yano model with optimized proportion of invariable sites) were used for construction of, respectively, NA and HA maximum-likelihood trees, as determined in jModelTest. aLRT (SH-like) branch support values equal or higher to 0.75 are indicated in the tree. Virus strains are symbol coded according to their phenotypic outlier classification: \#\#\# upper extreme outlier; \# upper mild outlier; * lower

a similar $\mathrm{IC}_{50}$ fold-change reduction. Both results support an over all difference between the oseltamivir resistance levels of the $\mathrm{H} 275 \mathrm{Y}$ viruses from the two $\mathrm{A}(\mathrm{H} 1 \mathrm{~N} 1)$ lineages. In addition to the NA H275Y mutation, genotypic analysis of A/Portugal/03/2011 identified HA N156D as the only other specific mutation of this virus strain. HA mutations at residue 156 $(\mathrm{N} 156 \mathrm{D} / \mathrm{S} / \mathrm{K} / \mathrm{T})$ had been described in $\mathrm{A}(\mathrm{H} 1 \mathrm{~N} 1) 2009$ mild outlier (colored web version: virus strains are colour coded according to their phenotypic outlier classification - red: upper extreme outlier; orange: upper mild outlier; green: lower mild outlier; black: non-outlier). When a virus strain was outlier for both NAIs but at different categories, the category associated with a higher IC50 fold-change was used for strain coding. Additionally, all strains from 2010/2011 are coloured in grey (colored web version: all strains from 2010/2011 are marked with the symbol \#). Reference virus strains are indicated in bold and uppercase and A/California/07/2009 vaccine strain is additionally in italic. All indicated cluster defining amino acid mutations were identified against vaccine strain sequence. HA established genetic clades are based in [WHO, 2012].

variant as commonly but not exclusively associated with virus propagation in MDCK-SIAT1 cells [WHO, 2011b]. In fact, another polymorphism at this residue (N156K) was observed in this study for one upper mild outlier. HA sequencing from the clinical specimen will be essential for confirmation. Also, residue 156 is located in the antigenic site $\mathrm{Sa}$ and therefore N156D mutation could be associated with the key antigenic 
role of HA. However, a potential compensatory role for the H275Y fitness deficit cannot be ruled out and could be tested through in vitro replication kinetics assays using wild-type and mutant viruses (without N156D mutation). HA sequencing is important for the study of antiviral drug susceptibility not only due to the potential compensatory role that HA mutations may have but essentially due to the direct impact that HA mutations can have on virus susceptibility. It is wellknown that HA and NA proteins work in concert during viral entry and viral release from the cell. Thus, if an HA mutation decreases the virus affinity for binding cell receptors, then it could also reduce the virus NA activity requirement for viral release and consequently reduce the virus susceptibility to any NAI [Zambon and Hayden, 2001].

The identification of the two H275Y oseltamivirresistant cases is in agreement with the sporadic detection of resistant cases that has been observed worldwide. Oseltamivir-resistant cases have emerged mainly as a result of antiviral drug use and immunosuppressive conditions, both criteria present for suspected case 2 [WHO, 2011a].

The presence of $\mathrm{H} 275 \mathrm{Y}$ resistant marker was not detected in the original specimen from clinical suspected resistant case 1 . Therefore, the evidence of clinical failure observed can be most probably a result of the high delay between the onset of symptoms and the start of antiviral therapy (11 days).

Other finding of this study was the identification of I223V mutation in the NA sequence of two $A(H 1 N 1)$ pdm09 virus strains exhibiting higher $\mathrm{IC}_{50}$ values to oseltamivir (three to four fold-change increase) and, to a lesser extent, to zanamivir ( $\sim 2$-fold-change increase).These virus strains are from two $\mathrm{A}(\mathrm{H} 1 \mathrm{~N} 1)$ pdm09 community cases from the 2009 pandemic period. NA I223V mutation was previously associated to a similar decreased susceptibility pattern in N1 viruses but only in one reverse genetic study [Nguyen et al., 2012]. During surveillance activities or clinical studies, this mutation was found in viruses with reduced susceptibility to oseltamivir (fold-change increase $>10)$ in the $\mathrm{A}(\mathrm{H} 3 \mathrm{~N} 2)$ and $\mathrm{B}$ subtype/type [Nguyen et al., 2012]. The clinical impact of I223V, as of most NAIs reduced susceptibility-conferring mutations, is still unclear. But, mutations at position 223 have previously been associated with clinical NAIs treatment failure [van der Vries et al., 2012]. No epidemiological link was known for the two patients harboring the NA I223V viruses, but the identical NA and HA sequences of the viruses and the temporal and geographic proximity of the cases, indicates a potential relation.

The observed lack of impact of NA Y155H mutation (HRI NAIs phenotype in $\mathrm{A}(\mathrm{H} 1 \mathrm{~N} 1)$ seasonal viruses), in the NAIs phenotypic profile of $\mathrm{A}(\mathrm{H} 1 \mathrm{~N} 1) \mathrm{pdm} 09$ viruses can be explained by structural differences on the NA of $\mathrm{A}(\mathrm{H} 1 \mathrm{~N} 1)$ pandemic and seasonal variants. These structural differences could also explain the significantly lower $\mathrm{IC}_{50}$ values exhibited by the virus- es from the pandemic variant. The absence of the 150-cavity, characteristic of $\mathrm{N} 1$ virus, in $\mathrm{A}(\mathrm{H} 1 \mathrm{~N} 1)$ pdm09 viruses was initially indicated as the main structural difference [ $\mathrm{Li}$ et al., 2010]. However, a recent study showed the presence of this cavity in all $\mathrm{N} 1$ and, surprisingly, in $\mathrm{N} 2$ viruses, raising new questions about NA structures [Amaro et al., 2011].

No genetic mechanism was found for the $\mathrm{IC}_{50}$ minor increase (1.4- to 2.1-fold-change) observed for most of oseltamivir and zanamivir upper outlier virus strains. No shared mutation was identified and approximately half of these virus strains lacked any specific mutations at NA or HA sequences. Additionally, the majority of the specific mutations identified probably derived from the key antigenic role of these two glycoproteins, given their location in antigenic sites or by creating potential $\mathrm{N}$-glycosylation sites that may prevent accessibility and recognition of antigenic sites by antibodies [Vigerust et al., 2007]. Other specific mutations, positioned in the stalk region of the protein, are most likely not related with antiviral susceptibility. Whole genome sequencing of upper outlier strains is being performed in order to find if mutations located in the other genome segments can be contributing for the $\mathrm{IC}_{50}$ minor increases observed. The understanding of these minor increases is one of the major current challenges in influenza antiviral drug susceptibility evaluation.

$\mathrm{A}(\mathrm{H} 1 \mathrm{~N} 1)$ pdm09 viruses were more susceptible to zanamivir than to oseltamivir. This drug-specific variation is characteristic of $\mathrm{N} 1$ viruses and results from differences in the intrinsic chemical properties of the two NAIs [Ferraris and Lina, 2008]. The chemical similarity that zanamivir exhibits to the natural substrate of NA (sialic acid) could explain the different phenotypic results obtained for this drug [van der Vries et al., 2012]. This includes the lack of significant differences between the $\mathrm{IC}_{50}$ values from the two time periods analyzed and the lower impact of NA I223V mutation on virus susceptibility (lower $\mathrm{IC}_{50}$ fold-change). Regarding oseltamivir, the two NA-specific mutations of 2010/2011 virus strains -N369K and V241I, may have contributed for the significantly higher $\mathrm{IC}_{50}$ values obtained during that season. Computational analysis showed that both mutations improve NA stability of H275Y oseltamivir-resistant virus and experimental studies showed that N369K increases NA surface expression and activity of this resistant variant [Hurt et al., 2012]. However, the unequal size of the two time period datasets $(\mathrm{N}=116$ for the pandemic period and $\mathrm{N}=26$ for 2010/2011) may also be contributing for this $\mathrm{IC}_{50}$ temporal difference.

The results obtained in this study may contribute to a better understanding of the currently challenging phenotype-genotype relationship. In addition, all national data presented here may contribute to the global assessment of $\mathrm{A}(\mathrm{H} 1 \mathrm{~N} 1)$ pdm09 virus susceptibility profile and baseline level to NAIs. 


\section{ACKNOWLEDGMENTS}

This work was supported by a research grant from the Portuguese Calouste Gulbenkian Foundation and by doctoral grants from the Portuguese Science and Technology Foundation (SFRH/BD/48532/2008, SFRH/BD/62676/2009, and SFRH/BD/65211/2009). These two funding sources had no involvement on the work. The authors would like to thank to the Technology and Innovation Unit of the National Health Institute Doutor Ricardo Jorge, IP (Lisbon, Portugal) for performing all automated sequencing reactions. The authors would also like to thank to the Public Health England (Colindale, London, UK), namely to Dr. Angie Lackenby, for allowing the carrying out of quasi-species quantification and for all the support given, including English revision of the manuscript.

\section{REFERENCES}

Amaro RE, Swift RV, Votapka L, Li WW, Walker RC, Bush RM. 2011. Mechanism of 150-cavity formation in influenza neuraminidase. Nat Commun 2:388.

AMC. 2001. Robust statistics: A method of coping with outliers. AMC Technical Brief, No. 6. http://www.rsc.org/images/brief6 tcm18-25948.pdf [accessed July 9, 2008].

CDC. 2009. Oseltamivir-resistant 2009 pandemic influenza A (H1N1) virus infection in two summer campers receiving prophylaxis-North Carolina, 2009. Morb Mortal Wkly Rep 58:969-972. http://www.cdc.gov/mmwr/pdf/wk/mm5835.pdf.

Correia V, Rebelo-de-Andrade H, Santos LA, Lackenby A, Zambon M. 2010. Antiviral drug profile of seasonal influenza viruses circulating in Portugal from 2004/2005 to 2008/2009 winter seasons. Antiviral Res 86:128-136.

Darriba D, Taboada GL, Doallo R, Posada D. 2012. jModelTest 2: More models, new heuristics and parallel computing. Nat Methods 9:772.

DGS. 2010. Relatório da pandemia da gripe em Portugal. Lisboa, Portugal: Direcção Geral da Saúde, Ministério da Saúde. http:// www.portaldasaude.pt/NR/rdonlyres/9BA67AE6-6A49-4B8DAC96-3E48B67A07A3/0/RelatorioPandemiaGripeAH1N12009Portugal.pdf [accessed February 11, 2013].

Dominguez-Cherit G, Lapinsky SE, Macias AE, Pinto R, EspinosaPerez L, de la Torre A, Poblano-Morales M, Baltazar-Torres JA, Bautista E, Martinez A, Martinez MA, Rivero E, Valdez R, RuizPalacios G, Hernandez M, Stewart TE, Fowler RA. 2009. Critically ill patients with 2009 influenza A(H1N1) in Mexico. JAMA 302:1880-1887.

ECDC. 2009. First isolation of a secondary oseltamivir-resistant A(H1N1)v strain in Denmark—July 1, 2009. http://ecdc.europa. $\mathrm{eu} / \mathrm{en} /$ healthtopics/documents/0906 influenza_ah1n1_ecdc threat assessment first isolation_of a secondary oseltamivir_resistant strain_in_denmark.pdf [accessed February 11, 2013].

Ferraris $\overline{\mathrm{O}}$, Lina B. 2008. Mutations of neuraminidase implicated in neuraminidase inhibitors resistance. J Clin Virol 41:13-19.

Giria MT, Rebelo-de-Andrade H, Santos LA, Correia VM, Pedro SV, Santos MA. 2012. Genomic signatures and antiviral drug susceptibility profile of A(H1N1)pdm09. J Clin Virol 53:140-144.

Guindon S, Gascuel O. 2003. A simple, fast, and accurate algorithm to estimate large phylogenies by maximum likelihood. Syst Biol 52:696-704.

HPA. 2006. Standard operating procedure for influenza MUNANA neuraminidase activity and inhibition assay (NO.V-66815). http:// www.isirv.org/site/images/stories/avg_documents/Methodology/ munana_ic50_sop_for_external.pdf [accessed July 11, 2012].

HPA. 2010. Standard operating procedure for pandemic 2009 H1N1 influenza A H275Y PCR and pyrosequencing (NO.V-6777). http:// www.isirv.org/site/images/stories/avg_documents/Methodology/wm v6777_pandemic\%202009\%20h1n1\%20influenza\%20a\%20h275y $\% 20$ pcr\%20and\%20pyrosequencing.pdf [accessed July 11, $2012]$.
Hurt AC, Hardie K, WilsoHurt AC, Hardie K, Wilson NJ, Deng YM, Osbourn M, Leang SK, Lee RT, Iannello P, Gehrig N, Shaw R, Wark P, Caldwell N, Givney RC, Xue L, Maurer-Stroh S, Dwyer DE, Wang B, Smith DW, Levy A, Booy R, Dixit R, Merritt T, Kelso A, Dalton C, Durrheim D, Barr IG. 2012. Characteristics of a widespread community cluster of $\mathrm{H} 275 \mathrm{Y}$ oseltamivirresistant A(H1N1)pdm09 influenza in Australia. J Infect Dis 206:148-157.

Igarashi M, Ito K, Yoshida R, Tomabechi D, Kida H, Takada A. 2010. Predicting the antigenic structure of the pandemic (H1N1) 2009 influenza virus hemagglutinin. PLoS ONE 5:e8553. DOI: 10.1371/journal.pone.0008553

Jain S, Kamimoto L, Bramley AM, Schmitz AM, Benoit SR, Louie J, Sugerman DE, Druckenmiller JK, Ritger KA, Chugh R, Jasuja S, Deutscher M, Chen S, Walker JD, Duchin JS, Lett S, Soliva S, Wells EV, Swerdlow D, Uyeki TM, Fiore AE, Olsen SJ, Fry AM, Bridges CB, Finelli L. 2009. Hospitalized patients with 2009 H1N1 influenza in the United States, April-June 2009. N Engl J Med 361:1935-1944.

Lackenby A, Moran Gilad J, Pebody R, Miah S, Calatayud L, Bolotin S, Vipond I, Muir P, Guiver M, McMenamin J, Reynolds A, Moore C, Gunson R, Thompson C, Galiano M, Bermingham A, Ellis J, Zambon M. 2011. Continued emergence and changing epidemiology of oseltamivir-resistant influenza A(H1N1)2009 virus, United Kingdom, winter 2010/11. Euro Surveill 16: pii=19784. http://www.eurosurveillance.org/ViewArticle.aspx? ArticleId $=19784$

Li Q, Qi J, Zhang W, Vavricka CJ, Shi Y, Wei J, Feng E, Shen J, Chen J, Liu D, He J, Yan J, Liu H, Jiang H, Teng M, Li X, Gao GF. 2010. The 2009 pandemic H1N1 neuraminidase N1 lacks the 150-cavity in its active site. Nat Struct Mol Biol 17:12661268.

Maurer-Stroh S, Ma J, Lee RT, Sirota FL, Eisenhaber F. 2009. Mapping the sequence mutations of the 2009 H1N1 influenza A virus neuraminidase relative to drug and antibody binding sites. Biol Direct 4:18. DOI: $10.1186 / 1745-6150-4-18$.

Meijer A, Lackenby A, Hay A, Zambon M. 2007. Influenza antiviral susceptibility monitoring activities in relation to national antiviral stockpiles in Europe during the winter 2006/2007 season. Euro Surveill 12:pii=698. http://www.eurosurveillance.org/ViewArticle.aspx?ArticleId $=698$.

Nguyen HT, Fry AM, Gubareva LV. 2012. Neuraminidase inhibitor resistance in influenza viruses and laboratory testing methods. Antivir Ther 17:159-173.

Oshitani H, Kamigaki T, Suzuki A. 2008. Major issues and challenges of influenza pandemic preparedness in developing countries. Emerg Infect Dis 14:875-880.

Pada S, Tambyah PA. 2011. Overview/reflections on the 2009 H1N1 pandemic. Microbes Infect 13:470-478.

Santos LA, Correia V, Gíria M, Pedro S, Santos MM, Silvestre MJ, Rebelo-de-Andrade H. 2011. Genetic and antiviral drug susceptibility profiles of pandemic $\mathrm{A}(\mathrm{H} 1 \mathrm{~N} 1) \mathrm{v}$ influenza virus circulating in Portugal. Influenza Other Respir Viruses 5:294-300.

Takashita E, Ejima M, Itoh R, Miura M, Ohnishi A, Nishimura H, Odagiri T, Tashiro M. 2014. A community cluster of influenza A(H1N1)pdm09 virus exhibiting cross-resistance to oseltamivir and peramivir in Japan, November to December 2013. Euro Surveill 19:pii=20666. http://www.eurosurveillance.org/images/ dynamic/EE/V19N01/art20666.pdf.

van der Vries E, Collins PJ, Vachieri SG, Xiong X, Liu J, Walker PA, Haire LF, Hay AJ, Schutten M, Osterhaus AD, Martin SR, Boucher CA, Skehel JJ, Gamblin SJ. 2012. H1N1 2009 pandemic influenza virus: Resistance of the I223R neuraminidase mutant explained by kinetic and structural analysis. PLoS Pathog 8: e1002914. DOI: 10.1371/journal.ppat.1002914

Vigerust DJ, Ulett KB, Boyd KL, Madsen J, Hawgood S, McCullers JA. 2007. N-linked glycosylation attenuates H3N2 influenza viruses. J Virol 81:8593-8600.

Wei H. 2010. The interaction between the 2009 H1N1 influenza A hemagglutinin and neuraminidase: Mutations, co-mutations, and the NA stalk motifs. J Biomed Sci Eng 3:1-12. DOI: 10.4236/jbise. 2010.31001

WHO. 2005. WHO checklist for influenza pandemic preparedness planning. http://www.who.int/influenza/resources/documents/FluCheck6web.pdf [accessed February 6, 2013].

WHO. 2009a. Pandemic influenza preparedness and response: A WHO guidance document. http://whqlibdoc.who.int/publications/ 2009/9789241547680_eng.pdf [accessed February 6, 2013]. 
WHO. 2009b. Sequencing primers and protocol. http://www.who.int/ csr/resources/publications/swineflu/GenomePrimers 20090512.pdf [accessed June 6, 2009].

WHO. 2010. Update on oseltamivir-resistant pandemic A (H1N1) 2009 influenza virus: January 2010. Wkly Epidemiol Rec 85:3740. http://www.who.int/wer/2010/wer8506.pdf.

WHO. 2011a. Global monitoring of antiviral resistance in currently circulating human influenza viruses, November 2011. Wkly Epidemiol Rec 86:497-501. http://www.who.int/wer/2011/wer8645.pdf.

WHO. 2011b. Report prepared for the WHO annual consultation on the composition of influenza vaccine for the Southern Hemisphere-September 26-30, 2011. WHO Collaborating Centre for Reference and Research on Influenza, National Institute for
Medical Research, London, United Kingdom. http://www.nimr mrc.ac.uk/documents/about/interim-report-sep-2011.pdf [accessed February 6, 2013].

WHO. 2012. Meetings of the WHO working group on surveillance of influenza antiviral susceptibility-Geneva, November 2011 and June 2012. Wkly Epidemiol Rec 87:369-374. http://www.who.int/ wer/2012/wer8739.pdf.

Yang H, Carney P, Stevens J. 2010. Structure and receptor binding properties of a pandemic H1N1 virus hemagglutinin. PLoS Curr 2:RRN1152. DOI: 10.1371/currents.RRN1152

Zambon M, Hayden FG. 2001. Position statement: Global neuraminidase inhibitor susceptibility network. Antiv Res 49 147-156. 